\title{
Visual Design and Anthropomorphism in a Mobile Pulmonary Rehabilitation Support Intervention
}

\author{
Cindy CHONG ${ }^{\text {a }}$, Danielle LOTTRIDGE ${ }^{\mathrm{a}}$, Jim WARREN ${ }^{\mathrm{a}, 1}$ and Rosie DOBSON ${ }^{\mathrm{b}}$ \\ ${ }^{a}$ School of Computer Science, University of Auckland \\ ${ }^{b}$ National Institute for Health Innovation, University of Auckland
}

\begin{abstract}
Pulmonary rehabilitation is a behavioral intervention that can improve symptom control and quality of life for patients with chronic obstructive pulmonary disease (COPD), but access, uptake and adherence are problematic. Our team has pursued the development of a mobile phone-based intervention (mobile pulmonary rehabilitation, $\mathrm{mPR}$ ) with iterative design and a pilot study. The $\mathrm{mPR}$ intervention is delivered through two technologies: text messages (SMS) and a smartphone application. Our user-centered design analysis of pilot study data led to several insights. First, patients' replies to the SMS suggested that messages were anthropomorphised and provided social support. Second, the smartphone application could help patients by clearly visualizing the exercise program, alternative exercises, and progress to date. We demonstrate the design iterations made to meet these requirements and we present feedback obtained from experts and from four COPD patients. We discuss implications for the design of mobile pulmonary rehabilitation interventions.
\end{abstract}

Keywords. mobile applications, consumer health informatics, chronic disease management, human-computer interaction, user experience, mHealth

\section{Introduction}

One of the gold standard interventions for the ongoing management of chronic obstructive pulmonary disease (COPD) is pulmonary rehabilitation (PR) which comprises of a supervised, individually tailored, multidisciplinary program of exercise training, breathing retraining and improving patient knowledge through education. A systematic review of participation in physical activity programs, including PR, identified barriers including changing health status and lack of support and enablers including specific goals and social and professional support [1].

To increase the reach of pulmonary rehabilitation, the development of a mobile based intervention (mobile pulmonary rehabilitation, $\mathrm{mPR}$ ) has been pursued via a project initiated in mid-2018. Modern mobile devices ('smartphones') support multiple distinct modalities for interventions. The capability of text message (SMS) programs to promote healthy behaviors with personalised interventions is well established and includes smoking cessation [2] as well as concurrent reduction in multiple cardiac risk

${ }^{1}$ Corresponding Author, Jim Warren, School of Computer Science, University of Auckland, Auckland 1142, New Zealand; E-mail: jim@cs.auckland.ac.nz. 
factors for patients with coronary heart disease [3]. Health promoting apps can include images and video, collect feedback with sensors (from the phone or other devices), provide graphical feedback on progress and support diaries [4, 5]. Health promoting interventions using mobile technology can facilitate rehabilitation in a variety of straightforward ways: through reminders and provision of easily accessible informational resources such exercises based on individual health status. Decades of experimental social science indicates that in many circumstances, people relate to computers in essentially social ways similar to the ways they relate to other people [6] (aka anthropomorphizing the system) As such, we suggest that mobile interventions can also offer something that might be unexpected from a technology: social support.

In this paper we present work on improving the user experience of the app component of a combined SMS and app based mPR intervention, based on identifying complementary roles of the SMS and app channels.

\section{Original mPR Design, Intervention and Pilot Field Test}

A mixed-methods (survey and interview) study undertaken for this project found high interest in $\mathrm{mPR}$ from both patients and healthcare professionals [7]. While participants saw the potential to improve access to PR through technology, there was concern about access to technology and digital literacy, as well as patient safety and the lack of group environment (as compared to gold standard PR). Patients and healthcare professionals identified key features for a mPR intervention, leading to the development of an $\mathrm{mPR}$ intervention with both personalised and tailored SMS and mobile app components.

The SMS intervention spanned 9 weeks; one core message per day provided motivation, support and information. There was also a weekly SMS exercise prescription message (at one of three baseline exercise capacity levels as defined by the enrolling PR clinician), smoking cessation support for smokers, and a weekly message on airway clearance for those with high secretion load. There was an optional family/ whānau version of the messages for those supporting the patient. Messages were personalised and tailored with the person's name, culturally specific greeting and names of any support people that were added. Example SMS messages include:

- Motivation: "mPR: [hi]. By doing the mPR program you can improve balance, muscle strength, general fitness, \& general wellbeing. We are here to support you!"

- Support: "mPR: [hi] [name]. If there is something concerning you or your family about your health it's a good idea to discuss it with your doctor or nurse"

- Exercise information: "mPR: It is normal to feel a bit stiff after you first start to exercise as your muscles may not be used to the exercises. Keep going - the stiffness will ease"

The mPR app was an optional addition to the SMS. The app provided goal-setting, videos of people completing the prescribed exercises at the patient's baseline exercise capacity, self-assessments (questionnaire and 1-minute sit to stand test), and reminders. The app also included relaxation audio files and information for family for their support role. Figure 1 shows a selection of screens. 

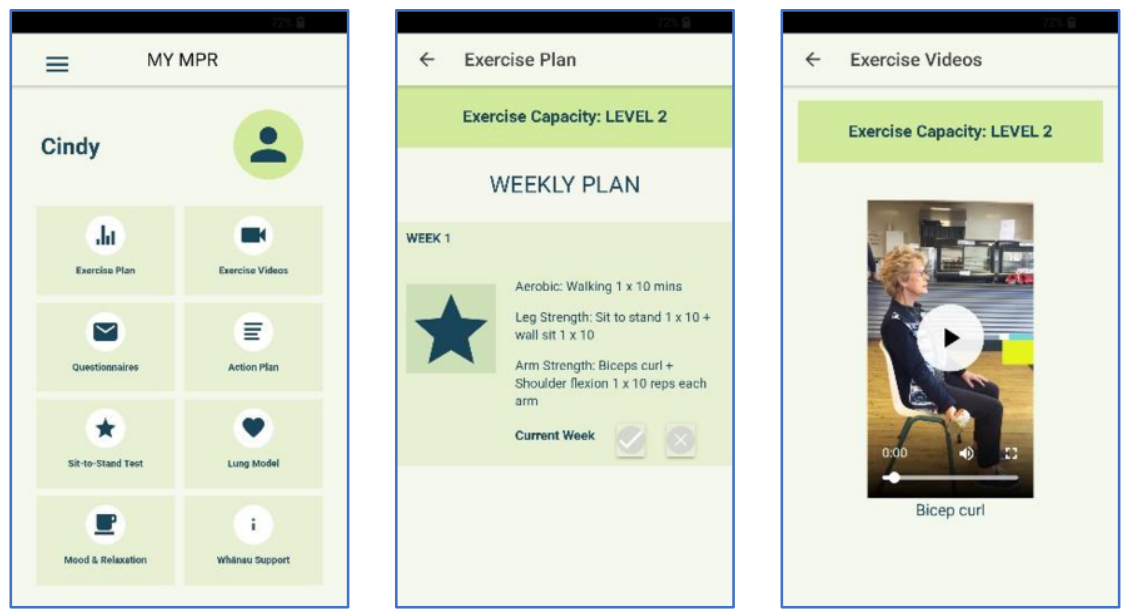

Figure 1. Screenshots of the initial mPR app that was deployed in the pilot study: (a) Dashboard screen; (b) Exercise Plan screen (c) Exercise Video screen.

A non-randomised pilot study of $\mathrm{mPR}$ was conducted between July and November 2019; 26 people with chronic respiratory disease who were eligible for PR participated, as well as 4 family members of participants. 18 participants (70\%) opted to use the app as well as SMS; of those, 11 signed-in to the app. 20 were available for follow-up interviews of whom 19 (95\%) said mPR helped them feel more supported, and 17 (85\%) said it helped them change their behavior. Full results are published elsewhere [8].

Of the 26 participants, 23 sent 390 SMS replies including exercise counts, health ratings, STOP messages, feedback messages and unexpected messages. Unexpected messages included updates: "Yup I do that and go shopping walking around supermarket" and "I am probably going to have a difficult time doing everything this week as in my car a lot!!" They asked questions such as "When I go home are you ok with me doing one walk in the water [pool] as causes no problems. Thanks." They made other remarkable responses: "[these messages are] what I've been looking for ages." and "Thank you for the support over the mPR program and thanks to all involved". There were 109 unexpected messages sent by 17 participants; 40 were Thank you messages, 11 were "Ok" and 6 were emoji message (sent from the same participant). The apparent anthropomorphizing of the SMS agent led us to note that SMS could act as social support to some participants, in addition to providing reminders and high-level information.

When patients were asked what they found most useful on the app, the most frequent response was the exercise videos. This was corroborated by examining the usage logs: exercise videos were viewed 88 times. When asked for suggestions of how the app could be improved, several patients mentioned they wanted to see their individual progress. Patients' feedback also led to requirements to add greater variability in exercises and simplify navigation. We noted that eight subsections of the application were given equal priority via equal screen real estate on the dashboard screen. In order to visually emphasise the most important functionality, we re-assigned screen space to functions that were most crucial and frequently accessed. 


\section{App Re-design}

We used a user-centered design approach [9] to reconsider app design requirements from subject matter experts, patients, and usage logs. We analyzed the functions delivered by the SMS intervention to determine requirements for the smartphone app. Together with the team, we confirmed that the highest priority of the smartphone app was to support access to information about the exercise program. Thus, the main needs for the app "home screen" would be high-level and related to the exercise program. Given the patients' user needs, design priorities, and the support provided through SMS, we designed a presentation of progress through the exercise program that could be holistically scanned "at a glance", i.e., glanceable. High-fidelity prototypes were created and shown to PR and m-Health experts for feedback. Comments were collated and edits were made to the prototype before re-showing the PR and m-Health experts to confirm the decisions in the re-design.

The re-design focused on three major areas: the home page, the exercise prescription page, and the videos page (Figure 2). No components of the app were removed. Components that were not prioritised were accessible from the menu. The prioritised focus of the app is exercise, which is why this component takes up approximately $80 \%$ of the home screen real estate (Figure 2a). The main screen has circles that fill in to represent progress towards completion of daily activity targets. There is a greyscale path through the exercises to provide patients with recommended ordering. The grey path was intentionally subtle to not divert visual emphasis from the exercises. Below the menu is a personalised welcome message to promote a feeling of personalisation. The bottom component of the main screen was prioritised for the other identified key elements that support COPD patients: breathing exercises, completion charts ("My progress") and what to do if feeling unwell. The menu on the top left includes the functions that were less used in the pilot study or were determined as not critical to the main functionality and focus of the app. These were self-assessment questionnaires, lung video, help documentation. Each exercise page included alternatives for the recommended exercise and accompanying videos (Figure 2b, 2c). When selected, the exercise videos open to a larger screen for easier viewing (Figure 2c). The green checkmark and green around the exercise icons indicates the patient's progress per day for each exercise (Figure 2a, 2b).

\section{Testing}

To evaluate the usability of the redesigned prototype, we sought feedback from clinicians and patients with usability testing methodology from user-centered design [9]. There were 4 patients, a nurse, a physiotherapist, and a doctor who agreed to take part. Two of the patients participated in the pilot study and so had familiarity with the earlier version of the app. User testing consisted of individual face to face sessions looking at a clickable prototype on a mobile phone, which was first demonstrated by the researcher and then handed over to the participant. A semi-structured interview format prompted discussion with asking about what was useful/liked and not useful/disliked and what other functions might be wanted. All participants were able to discover and view all parts of the app. Two out of four patients were positive about the app's overall usability with the comments the app "seemed easy to use" and "it looks like something I would use"; one was positive about components of the app and the fourth was neutral. 

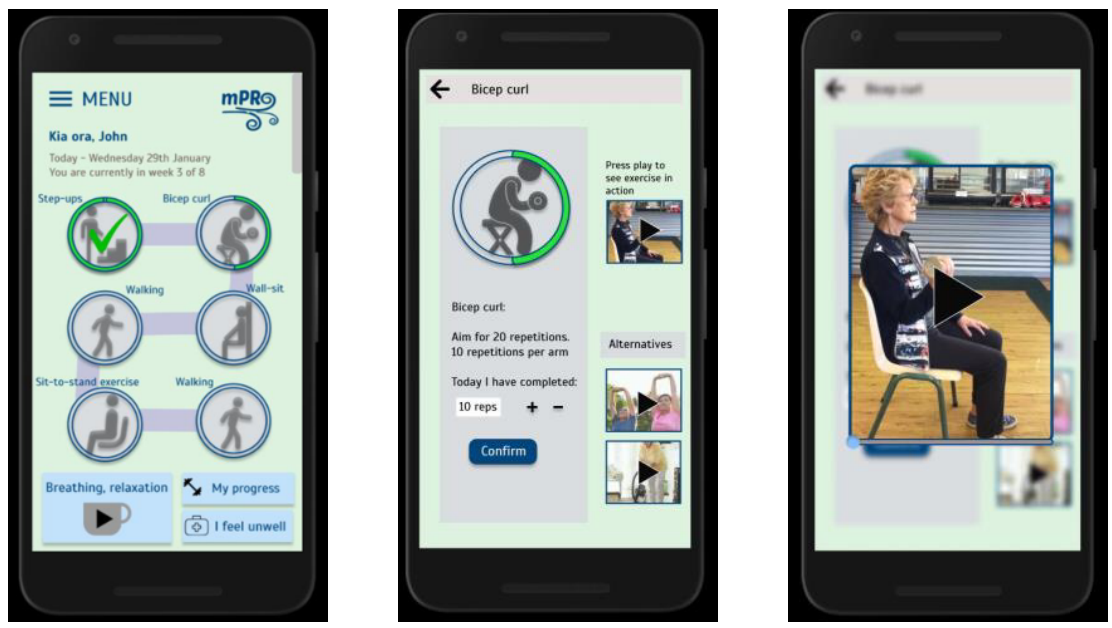

Figure 2. Screenshots of the re-designed mPR app based on design insights from the pilot study: (a) Home screen; (b) An exercise prescription screen; (c) Bicep curl video screen.

The exercise prescription and progress screens were well received. One patient stated: "This looks really good though, it looks like something I would use". Another stated: "Lets you know if you're slacking off sometimes, or maybe not going as well as you should for whatever reason." In Figure $2 b$, the grey path was intended to be a path which patients could follow to do exercises in a prescribed order; however, patients did not explore the exercises in this order. One of the clinicians did not understand the meaning of the path and thought it was "decoration," indicating the need to clarify further what is the 'next' exercise to be completed. No patient asked for clarification about the green progress circles, but one of the clinicians asked for clarification on them, indicating they may not be sufficiently emphasised or intuitive. The feedback received resulted in minor design revisions. We plan to use this redesign in an upcoming field trial.

\section{Discussion and Conclusion}

User-centered design and analysis applied to a mPR intervention resulted in insights related to complementary roles of the intervention's SMS and smartphone app technologies. The insights and user feedback led to substantial redesign of the app. The most notable aspect of the re-design was a glanceable presentation of the exercise program that incorporated progress. The main page of the re-designed app also prioritised other crucial features for COPD patients: e.g. what to do if acutely unwell. This approach is in line with Tendedez et al. [10] who found that patients with chronic respiratory conditions tended to use technology to manage their conditions reactively, such as recording peak flow readings or using their healthcare apps when they were feeling poorly and stopping self-managing or monitoring when they felt well.

In terms of future work, anthropomorphizing of the SMS was an insight from the user-centered design analysis; the strength of the social relationships and potential benefits or risks should be investigated. Further, there is potential for wearable 
technology and mobile sensors to track physical activity which would reduce patients' recording burden. Such monitoring technologies were not integrated with this app but will be investigated in future work. There are still basic barriers to use for smartphone apps; users had difficulties logging into the app. We encourage extensive evaluation testing of login to ensure this is not a barrier to use. More broadly, issues related to the relative isolation of patients when doing $\mathrm{mPR}$, such as safety in doing exercises as well as loss of social interaction, are an ongoing consideration. The most recent round of testing has obvious limitations of small sample size and limited user exposure to the app.

The combination of SMS-based interventions and a smartphone app is potentially powerful for delivering behavioral interventions such as pulmonary rehabilitation. SMS is more accessible due to simplicity of user interaction and, even in 2020, due to technical ubiquity (e.g. not everybody has a data plan even if they have a smartphone). However, smartphones offer features, such as providing videos and graphical feedback on progress, that can meaningfully expand the user experience of the intervention. This work has highlighted the value in understanding the relationship of these two mobile phone based modalities to guide app design, suggesting a surprisingly social role for SMS and a complementary informational, visual and progress-tracking roles for the smartphone app.

\section{Acknowledgements}

This work was funded by a Medtech CoRE grant. We thank the participants of the mPR pilot study, and the patients and clinicians at the pulmonary rehabilitation programs for their invaluable feedback. Thanks to Manoj Alwis for his feedback on design and implementation. Thanks to the mPR co-investigators and particularly Julie Reeve, Gayl Humphrey and Sarah Candy for their feedback on this manuscript.

\section{References}

[1] O. Thorpe, K. Johnston, and S. Kumar, "Barriers and enablers to physical activity participation in patients with COPD: a systematic review," J Cardiopulm Rehabil Prev, vol. 32, no. 6, pp. 359-69, Nov-Dec 2012.

[2] R. Whittaker, H. McRobbie, C. Bullen, A. Rodgers, Y. Gu, and R. Dobson, "Mobile phone text messaging and app-based interventions for smoking cessation," Cochrane Database Syst Rev, vol. 10, p. CD006611, Oct 222019.

[3] C. K. Chow et al., "Effect of Lifestyle-Focused Text Messaging on Risk Factor Modification in Patients With Coronary Heart Disease: A Randomized Clinical Trial," JAMA, vol. 314, no. 12, pp. 1255-63, Sep 22-29 2015.

[4] J. P. Higgins, "Smartphone Applications for Patients' Health and Fitness," Am J Med, vol. 129, no. 1, pp. 11-9, Jan 2016.

[5] K. L. Barnes, G. Dunivan, A. Jaramillo-Huff, T. Krantz, J. Thompson, and P. Jeppson, "Evaluation of Smartphone Pelvic Floor Exercise Applications Using Standardized Scoring System," Female Pelvic Med Reconstr Surg, vol. 25, no. 4, pp. 328-335, Jul/Aug 2019.

[6] B. Reeves and C. Nass, The media equation: How people treat computers, television, and new media like real people and places. Center for Study of Language and Information; Cambridge University Press, 1996.

[7] R. Dobson et al., "Understanding End-User Perspectives of Mobile Pulmonary Rehabilitation (mPR): Cross-Sectional Survey and Interviews," JMIR Form Res, vol. 3, no. 4, p. e15466, Dec 202019.

[8] R. Whittaker et al., "Mobile Pulmonary Rehabilitation (mPR): Pilot Study of a Prototype Mobile Phonebased Program," Frontiers in Computer Science, vol. under review, 2020.

[9] J. Preece, H. Sharp, and Y. Rogers, Interaction Design: Beyond Human-Computer Interaction, 4th ed. Wiley, 2015. 
[10] H. Tendedez, M. Ferrario, and R. McNaney, "Respiratory self-care: Identifying current challenges and future potentials for digital technology to support people with chronic respiratory conditions," in Proceedings of the 13th EAI International Conference on Pervasive Computing Technologies for Healthcare, 2019, pp. 129-138. 